\title{
Polymer-based dampening layer application to improve the operating shock tolerance of hard disk drive
}

\author{
Djati Wibowo Djamari ${ }^{*}$ (D), Fook Fah Yap ${ }^{2}$, Bentang Arief Budiman ${ }^{3}$ and Farid Triawan ${ }^{1}$
}

\author{
*Correspondence: djati.wibowo@ \\ sampoernauniversity.ac.id \\ ${ }^{1}$ Mechanical Engineering Study \\ Program, Sampoerna University, \\ Jakarta, Indonesia \\ Full list of author information is \\ available at the end of the article
}

\begin{abstract}
This paper discusses a passive vibration control method to improve the shock tolerance of hard disk drives (HDDs) in operating condition (op-shock tolerance). Past works in improving the HDDs' op-shock tolerance includes (i) parking the head when shock is detected, (ii) installing a lift-off limiter, (iii) structural modification of the suspension, and (iv) installing an external vibration isolation. Methods (i) and (iv) have practical issues, method (ii) works only on single shock direction, and method (iii) requires major engineering design/manufacturing work. Compared to these works, this paper proposes a method which has no practical issues and without requiring major engineering design/manufacturing work. The proposed method is to apply a polymer-based dampening layer on the backside of the baseplate with the purpose of increasing the damping ratio of the 1st bending mode of the baseplate. The location of the dampening layer on the baseplate is first determined by modal analysis and then fine-tuned by non-op-shock tests. The op-shock tolerance improvement is confirmed by op-shock tests where 2.5" HDD with the dampening layer on the baseplate can withstand a 300 G 0.5 -ms shock without failure while unmodified HDD can only withstand 250G 0.5-ms shock without failure.
\end{abstract}

Keywords: Hard disk drive, Shock tolerance, Baseplate mode, Damping, Polymer

\section{Introduction}

The demand for higher density hard disk drives (HDDs) pushes the requirements for the head-disk spacing. The greater the HDDs' density, the smaller the head-disk spacing required (see [1-3]). The head-disk spacing can be designed by setting the slider's flying height. Meanwhile, the flying height of the slider affects the stiffness of the air bearing, and more importantly, the shock response of the HDDs (see [4]). In operating condition, HDDs need to be protected from failures which are caused by external disturbance, i.e., external shock. Studies on HDDs' failure mechanism due to external shock can be found in $[5,6]$ and the references therein. HDDs fail when the head is touching the disk.

(c) The Author(s). 2022 Open Access This article is licensed under a Creative Commons Attribution 4.0 International License, which permits use, sharing, adaptation, distribution and reproduction in any medium or format, as long as you give appropriate credit to the original author(s) and the source, provide a link to the Creative Commons licence, and indicate if changes were made. The images or other third party material in this article are included in the article's Creative Commons licence, unless indicated otherwise in a credit line to the material. If material is not included in the article's Creative Commons licence and your intended use is not permitted by statutory regulation or exceeds the permitted use, you will need to obtain permission directly from the copyright holder. To view a copy of this licence, visit http://creativecommons.org/licenses/by/4.0/. The Creative Commons Public Domain Dedication waiver (http://creativecommons.org/publicdomain/zero/1.0/) applies to the data made available in this article, unless otherwise stated in a credit line to the data. 
Studies on HDDs' failure mechanism show that HDDs have specific op-shock resistance (see $[2,5,7])$. For example, there exists a range of external shock input amplitude and duration for which the head is not touching the disk. A common practice by HDD's manufacturer is to mention the op-shock tolerance of their product for a certain shock duration. An HDD having op-shock tolerance of 350 G 2 ms (milliseconds), where $1 \mathrm{G}=9.81 \mathrm{~m} / \mathrm{s}^{2}$, means that it can withstand external shock with a duration of 2 ms up to $350 \mathrm{G}$ of amplitude without failure. A study on shock duration effect to the shock response of HDD can be found in [8]. Generally, HDDs are more prone to failures from short shock duration.

There are various methods for protecting operating HDDs from failure. In a recent work by Nicholson et al. [9], the HDD is protected from external shock by parking the head when the HDD is subjected to shock. In parking position, where the disk can vibrate without touching the head, the HDD has relatively higher shock tolerance. However, the read/write performance of the HDD is sacrificed since it cannot perform its task in parking position. In the work of Ng et al. [10], the HDD is protected by installing a lift-off limiter. When the positive shock is high enough, the slider will move away from the disk and be separated from the air bearing (lift-off). This separation breaks the air bearing, and the sudden return of the slider makes the head touch the disk. This phenomenon is commonly called head-slap. The lift-off limiter prevents the slider from moving away from the disk to sustain the air bearing. However, the lift-off limiter can only work for one side of the disk during positive shock and the other side of the disk during negative shock.

Another method to protect HDDs from failure focus on modifying the HDDs' structure to improve the op-shock tolerance. In the work [5], a stiffer suspension design is proposed. On short shock duration of less than $2 \mathrm{~ms}$, stiffer suspension design works to increase the op-shock tolerance of the HDD. However, for a shock duration of $2 \mathrm{~ms}$ and longer, the stiffer suspension design has minimal effect. The work [2] focuses on HDD with a secondary stage actuator which used for fine control of track following in highdensity HDDs. HDD with secondary stage actuator has poor shock tolerance due to large mass at the tip. The work [2] proposes a secondary actuator design that has a lower mass without sacrificing the stroke sensitivity of the actuator. With a lower mass of secondary stage actuators, it is expected that the HDD with secondary stage actuators has better opshock tolerance. The work [11] proposes topology design optimization of suspension to improve HDD suspension dynamic characteristics. Although it is claimed that the optimized suspension design results in dynamic response improvement over shock input, the work does not study the op-shock tolerance improvement.

Another method to protect HDDs from failure rely on external shock isolation. The work [12] proposes a rubber mount design to isolate operating HDDs from shock and vibration. The rubber mounts work by reducing the shock energy transmitted to the HDD's baseplate which could result in higher op-shock tolerance. However, as studied by Djamari [13], the improvement to the op-shock tolerance by using rubber mounts is not significant when using minimum external footprint. Generally, an external shock isolation system needs a large footprint for it to be able to work effectively [14, 15]. Effective external shock isolation must have a relatively low dominant frequency and thus makes the isolated system vibrate with a large amplitude when subjected to a shock input. Recent work on external shock isolation of HDDs can be found in $[7,16,17]$. 
In summary, the op-shock tolerance of HDDs can be improved through the following: (i) intervention of HDD operation, (ii) design modification of the internal structure of HDDs, and (iii) installing an external shock isolation system. Method (i) improves the op-shock tolerance significantly, but it sacrifices HDD performance which is not practical. Method (ii) could potentially improve the op-shock tolerance, but there is a significant cost in changing the internal HDD structure design. Meanwhile, method (iii) offers little to no changes in HDD design, but it needs sufficient footprint which may not be practical at some point. All three methods solve the problem but are impractical which could limit the application of the HDDs. This paper proposes a method of external HDD shock isolation that neither changes the footprint of the standard HDD form factor nor change the HDD's design. It also does not sacrifice the HDD's performance and it works for both positive and negative shocks.

The proposed method in this paper is to apply a dampening layer (damper in a form of a thin polymer layer) on the backside of the baseplate with the purpose of increasing the damping ratio of the 1st bending mode of the baseplate, thus reducing the shock transmissibility to the HAA (Head Actuator Assembly). The application of damper to reduce the vibration of a structure is a common engineering solution. However, the damper location and how much damping that must be applied depend on the problem at hand and is not obvious. Recent work by Sezgen and Tinkir [18] shows that damper application is effective in reducing the vibration of a structure, and genetic algorithm is used to optimize the damper configuration. The work by Biglari et al. [19] shows that frictional damper can be used to reduce the residual vibration of a flexible manipulator, and several optimization methods are utilized to obtain the optimum structure of the damper. While Sezgen and Tinkir use mathematical model only to investigate the damper application, Biglari et al. use mathematical model and perform experimentation to test the optimized damper structure. Similar to these works, this paper also uses mathematical model of HDD to show the effectiveness of the damper, and similar to Biglari et al., this work perform experimentation to show the vibration reduction (nonoperating shock tests) after the damper is applied to the structure being studied. However, in HDD case, vibration reduction of the structure (obtained through nonoperating shock tests) needs to be verified with op-shock tests to show that damper application improves the op-shock tolerance. Therefore, in this work, the op-shock tests are done in addition to the non-operating shock test.

The rest of this paper is organized as follows. The "Methods" section discusses the problem statement and methodology. The "Results and discussion" section discusses theoretical background of the proposed method, MATLAB simulation of a simple HDD model, and experiment results for HDDs under non-operating and operating conditions. The "Conclusion" section concludes this paper.

\section{Methods}

The problem under consideration is a 2.5 " HDD in operating condition, with a single platter (see Fig. 1). The arm is positioned at the outer-disk position, which is the worst position due to large displacement of the disk at the outer-disk position compared to other arm's position that is closer to the disk-spindle when the HDD is subjected to shock. The problem in this paper is to design an external shock isolator for the HDD so that it can withstand higher op-shock without failure compared to the baseline 


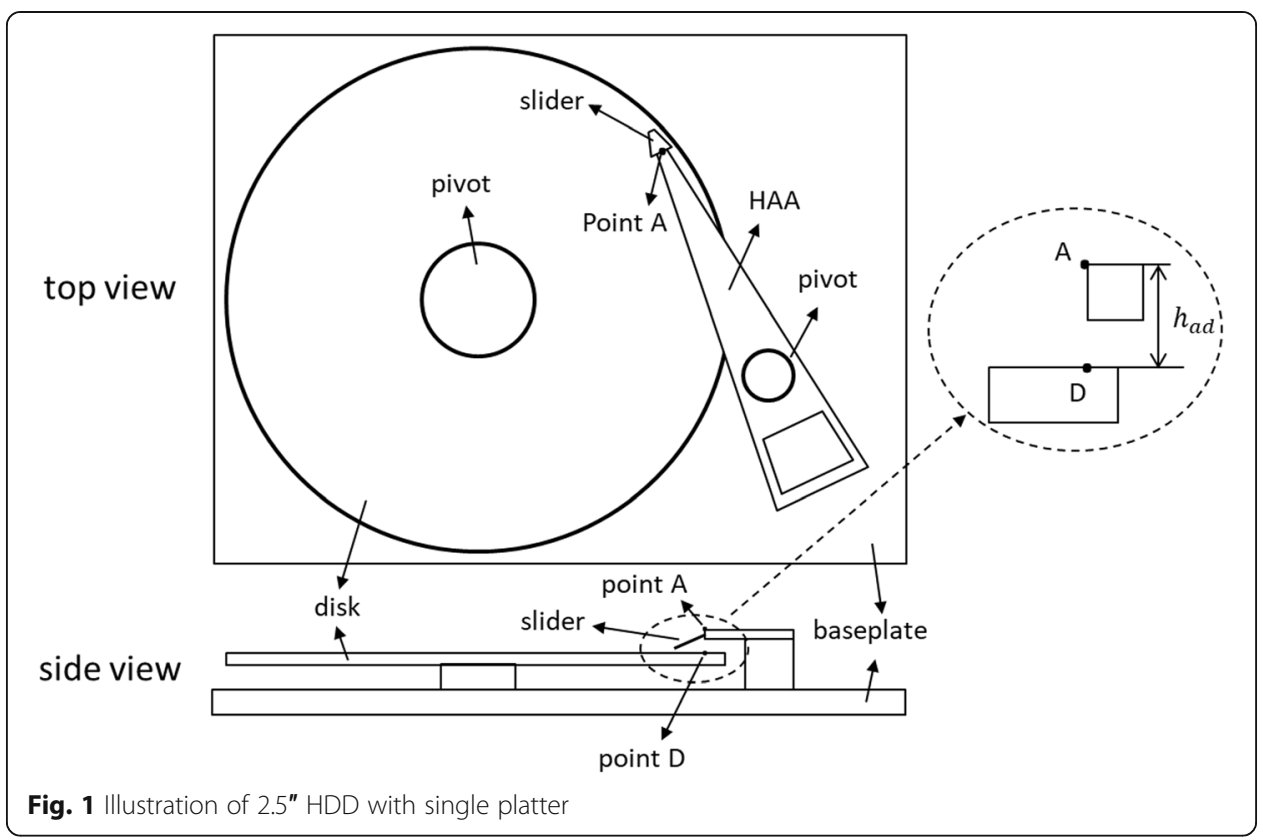

HDD. In this context, failure means the head touches the disk. As a design constraint, the external shock isolator must not change the form factor of the HDD.

We hypothesize that a reduction in the relative arm-disk displacement when the HDD is subjected to shock translates to the improvement of the op-shock tolerance. In other words, when the HDD structure is modified such that the relative arm-disk displacement is reduced, then the modified HDD can withstand higher op-shock without failure. To this end, let us consider Fig. 1 which illustrates the HDD structure. Let $h_{\text {ad }}$ be the vertical distance between the arm and the disk (the distance between point A and D, in Fig. 1). When the HDD is subjected to shock, i.e., the shock comes through the baseplate and then transmitted to the HAA and the disk, then $h_{a d}$ changes over time. If $h_{a d}$ becomes too small due to the shock (the arm becomes too close to the disk), the arm pushes the suspension towards the disk and the pushing force could break the air bearing which can make the head to touch the disk.

On the other hand, if $h_{a d}$ becomes too large due to the shock (the arm is moving away too far from the disk), the arm pulls the suspension away from the disk and the air bearing could also break due to too much force pulling the suspension away from the disk. The returning movement of the suspension can potentially result in head slap. Therefore, to reduce the risk of the head touching the disk, the changes in $h_{a d}$ must be kept as small as possible. The logic behind the above analysis is that when the head is not separated from the disk, the bending mode of the suspension is much higher than the bending mode of the arm. Thus, during the shock and before the separation between the head and the disk occurs, the suspension follows the movement of the arm. In conclusion, in this paper, we assume that the reduction in the changes of $h_{a d}$ when the HDD is subjected to shock implies the improvement in the op-shock tolerance of the HDD.

To reduce the changes in $h_{a d}$ when the HDD is subjected to shock, the proposed method in this paper is to apply a dampening layer to the backside of the baseplate, in between the baseplate and the PCB (Printed Circuit Board). The dampening layer is a 
thin polymer material that has high damping factor. This application increases the damping factor of the baseplate. The question is, how much and where we must apply the dampening layer?

Firstly, a theoretical analysis is done to mathematically show that increasing the damping factor of the baseplate can reduce the arm-disk relative displacement when the HDD is subjected to external shock. The theoretical analysis is done by modeling the baseplate, HAA, and disk using mass-spring-damper system. It will be shown that increasing the damping factor of the baseplate increases the damping ratio for all mode shapes and it reduces the changes in $h_{a d}$ when the HDD is subjected to shock.

The second step is to perform simulations on a simple model of HDD using MATLAB. The purpose is to find out the reduction in the changes of $h_{a d}$ for several shock input durations. It will also be shown that the application of damper will have a negative effect if too much damping factor is added to the baseplate. A comparison of reduction in the changes of $h_{a d}$ between application of damper on the baseplate and application of damper on the arm structure is also done to show that applying damper on the baseplate is more effective in reducing the changes in $h_{a d}$.

The next step is to define the areas on the baseplate where the dampening layer will be applied. For this, the non-op-shock tests are carried out for HDDs with and without the dampening layer, and the arm-disk relative displacement is measured by using fiber optic interferometer. The best dampening layer configuration is then used in the opshock tests to verify the op-shock tolerance improvement. In the tests, the dampening layer selection is not done based on the previous steps since the primary criterion for the dampening layer is its low outgassing property and it should be thin enough such that it does not affect the overall PCB assembly. Thus, for the tests, we use the available dampening layer product suitable for HDD application.

\section{Results and discussion}

\section{Theoretical analysis}

A model of baseplate-HAA-disk under consideration is shown in Fig. 2. The mass, stiffness, and damping coefficient of HAA are denoted by $m_{a}, k_{a}$, and $c_{a}$, respectively. The mass, stiffness, and damping coefficient of the disk are denoted by $m_{d}$, $k_{d}$, and $c_{d}$ respectively. The mass, stiffness, and damping coefficient of the baseplate are denoted by $m_{b}, k_{b}$, and $c_{b}$, respectively. Meanwhile, $m_{s}$ is the shaker mass and $F$ is the external force applied to shaker mass (note that $F$ is a function of time, $t$ ). The states $x_{a}, x_{d}, x_{b}$, and $x_{s}$ are the displacement of the arm tip, disk tip, baseplate, and the shaker, respectively. In the discussion in this section, we will see the effect of changing $c_{b}$ to the changes in $h_{a d}$. In our experiment that will be presented in subsections "Non-operating shock experiments" and "Operating shock experiments," $c_{b}$ is increased by applying a polymer-based dampening layer to the back of the baseplate. Due to relatively small mass and stiffness of the dampening layer compared to the mass and stiffness of the baseplate, in the analysis done in this section, we assume that the increase in $c_{b}$ does not affect the value of $m_{b}$ and $k_{b}$. 


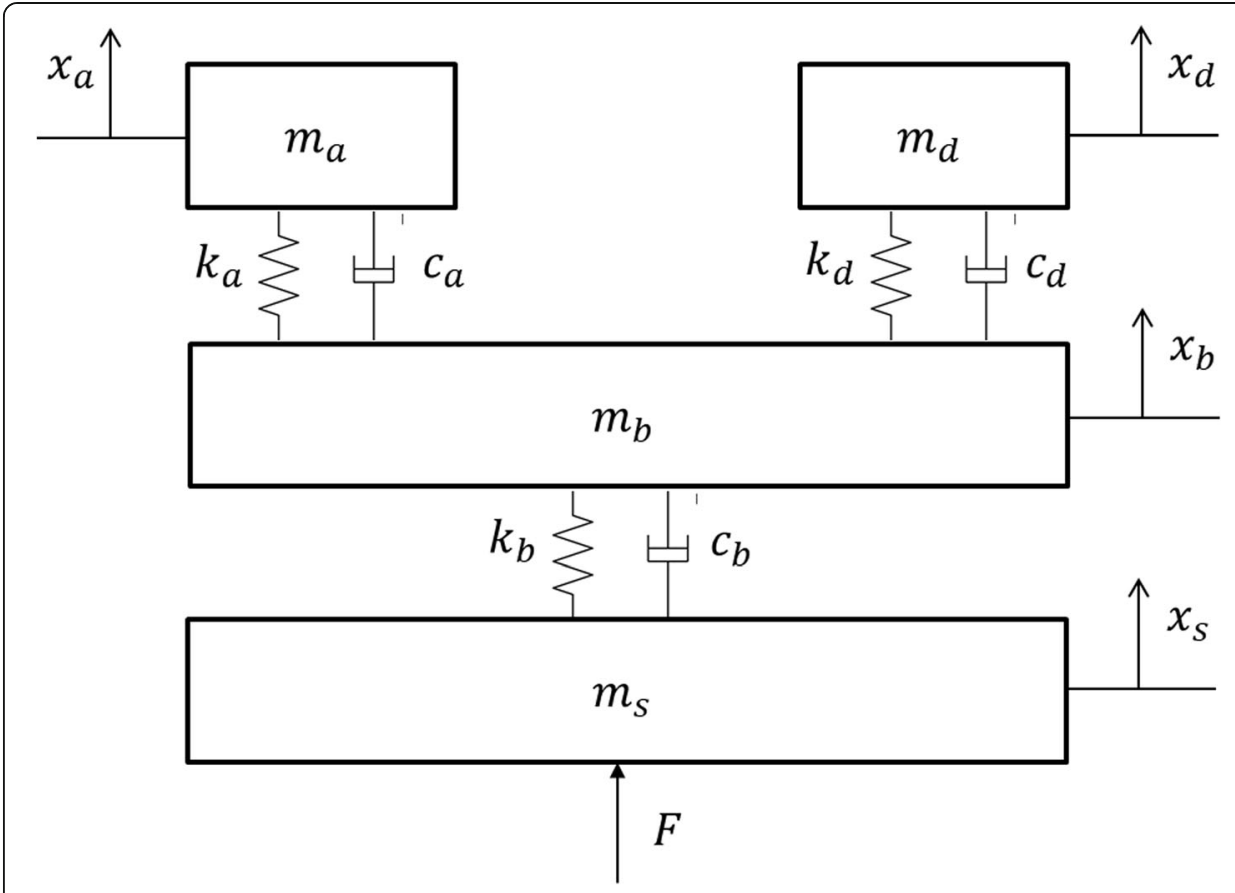

Fig. 2 A simple model of baseplate-HAA-Disk with shaker

The equation of motion of the model in Fig. 2 is the following:

$$
\begin{aligned}
& {\left[\begin{array}{cccc}
m_{s} & 0 & 0 & 0 \\
0 & m_{b} & 0 & 0 \\
0 & 0 & m_{a} & 0 \\
0 & 0 & 0 & m_{d}
\end{array}\right]\left[\begin{array}{l}
\ddot{x}_{s} \\
\ddot{x}_{b} \\
\ddot{x}_{a} \\
\ddot{x}_{d}
\end{array}\right]+\left[\begin{array}{cccc}
c_{b} & -c_{b} & 0 & 0 \\
-c_{b} & \bar{c} & -c_{a} & -c_{d} \\
0 & -c_{a} & c_{a} & 0 \\
0 & -c_{d} & 0 & c_{d}
\end{array}\right]\left[\begin{array}{l}
\dot{x}_{s} \\
\dot{x}_{b} \\
\dot{x}_{a} \\
\dot{x}_{d}
\end{array}\right]} \\
& +\left[\begin{array}{cccc}
k_{b} & -k_{b} & 0 & 0 \\
-k_{b} & \bar{k} & -k_{a} & -k_{d} \\
0 & -k_{a} & k_{a} & 0 \\
0 & -k_{d} & 0 & k_{d}
\end{array}\right]\left[\begin{array}{l}
x_{s} \\
x_{b} \\
x_{a} \\
x_{d}
\end{array}\right]=\left[\begin{array}{c}
F \\
0 \\
0 \\
0
\end{array}\right]
\end{aligned}
$$

where $\bar{c}=c_{a}+c_{b}+c_{d}$ and $\bar{k}=k_{a}+k_{b}+k_{d}$. Let $x=\left[\begin{array}{llll}x_{s} & x_{b} & x_{a} & x_{d}\end{array}\right]^{T}$, Eq. (1) can be compactly written as

$$
M \ddot{x}+C \dot{x}+K x=F_{v}
$$

where $M, C$, and $K$ are the mass matrix, damping matrix, and stiffness matrix, respectively.

Let $\dot{x}_{s}=v_{s}, \dot{x}_{b}=v_{b}, \dot{x}_{a}=v_{a}, \dot{x}_{d}=v_{d}$, and $v=\left[\begin{array}{llll}v_{s} & v_{b} & v_{a} & v_{d}\end{array}\right]^{T}$, the state space equation of the simplified model is given by:

$$
\left[\begin{array}{c}
\dot{x} \\
\dot{v}
\end{array}\right]=\left[\begin{array}{cc}
0_{4} & I_{4} \\
-M^{-1} K & -M^{-1} C
\end{array}\right]\left[\begin{array}{l}
x \\
v
\end{array}\right]+\left[\begin{array}{c}
0 \\
M^{-1}
\end{array}\right] F_{v}
$$

which can simply be written as

$$
\left[\begin{array}{c}
\dot{x} \\
\dot{v}
\end{array}\right]=A\left[\begin{array}{l}
x \\
v
\end{array}\right]+B F_{v}
$$


where $A$ is the state matrix and $B$ is the input matrix. Assuming nonzero damping with underdamped condition, then the eigenvalues of the state matrix can be expressed as $\left\{0,0,-p_{2} \pm j \omega_{d_{2}},-p_{3} \pm j \omega_{d_{3}},-p_{4} \pm j \omega_{d_{4}}\right\}$, where $p_{4}=\zeta_{4} \omega_{n_{4}}, p_{3}=\zeta_{3} \omega_{n_{3}}$, and $p_{2}=\zeta_{2}$ $\omega_{n_{2}}$ are the real part of the eigenvalues, $\omega_{d_{2}}=\omega_{2} \sqrt{1-\zeta_{2}^{2}}, \omega_{d_{3}}=\omega_{3} \sqrt{1-\zeta_{3}^{2}}$, and $\omega_{d_{4}}$ $=\omega_{4} \sqrt{1-\zeta_{4}^{2}}$ are the imaginary part of the eigenvalues or the damped natural frequencies. The first two zero eigenvalues correspond to the rigid body mode of all masses or the 1 st mode of the system. In this setting, $\zeta_{2}, \zeta_{3}$, and $\zeta_{4}$ are the damping ratio for the 2nd, 3rd, and 4th modes, respectively. The 2nd mode is the first bending mode of the baseplate, the 3rd mode is the first bending mode of the disk, and the 4th mode is the first bending mode of the arm. Considering only the flexible modes, we know that the simple model is a stable system, i.e., it will return to its equilibrium after it is disturbed temporarily (for example by knocking the arm tip). When the system is disturbed temporarily, the real part of the eigenvalues of matrix $A$ determines how fast the simple model return to the equilibrium, and the imaginary part determines the oscillation frequency of the response. For vibrating system, the convergence speed is represented by $\zeta \omega_{n}$, and the oscillation of the response is represented by the $\omega_{d}$.

It can be shown by parametric study, by inserting values and varying the variables (since the closed form solution of the eigenvalues of matrix $A$ is not possible to be shown), that the damping factor of the baseplate, $c_{b}$, affects the damping ratio of all nonzero modes. Meanwhile, the damping factor of the disk, $c_{d}$, dominantly affects only the 3rd mode, and the damping factor of the arm, $c_{a}$, dominantly affects only the damping ratio of the 4th mode. This is due to the coupling between the baseplate and the arm-disk. Meanwhile, the arm is not coupled to the disk.

The force $F$ is a shock input which models the impact when HDD is dropped. To show the effectiveness of increasing the baseplate's damping factor in minimizing the changes of $h_{a d}$, we assume that $F$ is an impulse and thus to obtain the solution of (2), we assume initial state $v_{s}(0)>0$, while the rest of the initial states being zero and $F$ is set to be zero. Let $L=\left[\begin{array}{lll}l_{1} & \cdots & l_{8}\end{array}\right]$ with $l_{i}=\left[\begin{array}{lll}l_{i 1} & \cdots & l_{i 8}\end{array}\right]^{T}$ for $i=1, \cdots, 8$ be the left eigenvector of the state matrix, where $q^{T}$ is the transpose of $q$, and $R=\left[\begin{array}{lll}r_{1} & \cdots & r_{8}\end{array}\right]$ with $r_{i}=\left[\begin{array}{lll}r_{i 1} & \cdots & r_{i 8}\end{array}\right]^{T}$ for $i=1, \cdots, 8$ be the right eigenvector of the state matrix, the solution to (2) can be expressed as

$$
\left[\begin{array}{l}
x(t) \\
v(t)
\end{array}\right]=e^{A t}\left[\begin{array}{c}
x(0) \\
v(0)
\end{array}\right]=R e^{\Lambda t} L\left[\begin{array}{c}
x(0) \\
v(0)
\end{array}\right]
$$

where $\Lambda$ is a diagonal matrix of the eigenvalues of matrix $A$ :

$$
\begin{aligned}
\Lambda & =\operatorname{diag}\left\{\lambda_{1}, \lambda_{2}, \cdots, \lambda_{8}\right\} \Lambda \\
& =\operatorname{diag}\left\{0,0,-p_{2}+j \omega_{d_{2}},-p_{2}-j \omega_{d_{2}},-p_{3}+j \omega_{d_{3}},-p_{3}-j \omega_{d_{3}},-p_{4}+j \omega_{d_{4}},-p_{4}-j \omega_{d_{4}}\right\}
\end{aligned}
$$

Let $z=\left[\begin{array}{ll}x^{T} & v^{T}\end{array}\right]^{T}=\left[\begin{array}{lll}z_{1} & \cdots & z_{8}\end{array}\right]^{T}$, the solution to (2) can be written as follows:

$$
z_{i}(t)=\sum_{j=1}^{8} r_{j i} l_{1 j} e^{\lambda_{j} t} z_{1}(0)+\cdots+\sum_{j=1}^{8} r_{j i} l_{8 j} e^{\lambda_{j} t} z_{8}(0) ; i=1, \cdots, 8
$$

Since $z_{i}(0)=0$ for all $i$ except for $z_{5}(0)=v_{s}(0)>0$, we can write (5) as 


$$
z_{i}(t)=\sum_{j=1}^{8} r_{j i} l_{5 j} e^{\lambda_{j} t} z_{5}(0) ; i=1, \cdots, 8
$$

The relative displacement between the arm and the disk, $\delta_{a d}$, can be expressed as

$$
\begin{aligned}
\delta_{a d} & =z_{3}(t)-z_{4}(t)=\sum_{j=1}^{8} r_{j 3} l_{5 j} e^{\lambda_{j} t} z_{5}(0)-\sum_{j=1}^{8} r_{j 4} l_{5 j} e^{\lambda_{j} t} z_{5}(0) \delta_{a d} \\
& =z_{5}(0) \sum_{j=1}^{8}\left(r_{j 3}-r_{j 4}\right) l_{4 j} e^{\lambda_{j} t}
\end{aligned}
$$

If we want $h_{a d}$ to be as constant as possible, then it implies that $\delta_{a d}$ must be as small as possible. Clearly, if $\left(r_{j 3}-r_{j 4}\right)=0$ for all $j$, then $\delta_{a d}$ will be zero. However, this is not possible to happen. The most reasonable method in minimizing $\delta_{a d}$ is by minimizing the term $e^{\lambda_{j} t}$ for all $j$. The first two $\lambda^{\prime} s$ are zero due to the rigid body mode, so these cannot be changed. Meanwhile, the remaining six $\lambda^{\prime} s$ can be changed by modifying the spring and damper of the model. As we said earlier, changing $c_{b}$ allows us to change the damping ratio of the nonzero modes. Thus, the six $\lambda^{\prime} s$ will be significantly affected by $c_{b}$.

When we increase $c_{b}$, assuming that $\omega_{2}, \omega_{3}$, and $\omega_{4}$ are unchanged due to nonsignificant change in $m_{b}$ and $k_{b}$, then $\zeta_{2}, \zeta_{3}$, and $\zeta_{4}$ will be larger. This results in the smaller values of $\omega_{d_{2}}, \omega_{d_{3}}$, and $\omega_{d_{4}}$, which in turn reduces the oscillation frequency of the shock response. Other than that, due to larger $\zeta_{2}, \zeta_{3}$, and $\zeta_{4}$, then $\left|p_{2}\right|,\left|p_{3}\right|$, and $\left|p_{4}\right|$ will be larger or that the real part of nonzero eigenvalues will be more to the left of the imaginary axis. This results in a higher convergence rate of $\delta_{a d}$. On the other hand, if we only make the $c_{a}$ to be larger, i.e., only $\zeta_{4}$ is increased, then only $\omega_{d_{4}}$ will become smaller and only $\left|p_{4}\right|$ that will become larger. Thus, the convergence rate of $e^{\lambda_{3} t}$ and $e^{\lambda_{4} t}$ will still dominate the convergence of $\delta_{a d}$. This results in no to little improvement in the reduction of $\delta_{a d}$.

\section{Simulation of the simplified baseplate-HAA-disk model}

Typically for 2.5 " HDD, the first bending mode of the baseplate is around $600-800 \mathrm{~Hz}$, the first bending mode of the disk is around $1000-1100 \mathrm{~Hz}$, and the first bending mode of the arm is around 1500-1600 Hz (see [20]). To simulate the model in Fig. 2, we first define $m_{b}=35 \mathrm{~g}, m_{a}=2.8 \mathrm{~g}, m_{d}=5 \mathrm{~g}$, and $m_{s}=1 \cdot 10^{6} \mathrm{~g}$. These values are taken from the typical mass of the $2.5^{\prime \prime} \mathrm{HDD}$ and the shaker mass is defined for ease in defining the force input. Then, we tune $k_{b}=6.7153 \cdot 10^{5} \frac{\mathrm{N}}{\mathrm{m}}, k_{a}=2.2409 \cdot 10^{5} \frac{\mathrm{N}}{\mathrm{m}}$, and $k_{d}=$ $1.6581 \cdot 10^{6}$ such that the undamped-nonzero modes assume values around the typical modes of 2.5" HDD. The undamped-eigenpairs of the model simulated in this paper are:

$$
\begin{aligned}
& \gamma_{1}=0 H z, U_{1}=s_{1}\left[\begin{array}{llll}
1 & 1 & 1 & 1
\end{array}\right]^{T} \gamma_{2}=600 H z, U_{2}=s_{2}\left[\begin{array}{llll}
0 & 1 & 1.21 & 1.77
\end{array}\right]^{T} \gamma_{3} \\
& =1000 \mathrm{~Hz}, U_{3}=s_{3}\left[\begin{array}{llll}
0 & 1 & 1.97 & -4.71
\end{array}\right]^{T} \gamma_{4}=1500 \mathrm{~Hz}, U_{4} \\
& =s_{4}\left[\begin{array}{llll}
0 & 1 & -8.79 & -0.57
\end{array}\right]^{T}
\end{aligned}
$$

where $s_{i}$ for $i=1,2,3,4$ is a real number. The damping factor of the baseplate, arm, and disk is tuned such that the damping ratio for all modes is 0.01 . This value is a conservative damping ratio value of metals [21]. The damping factor of the baseplate, arm, 
and disk which results in this damping ratio is called as the unmodified configuration ( $\left.c_{b}^{0}=3.9584 \frac{\mathrm{Ns}}{\mathrm{m}}, c_{a}^{0}=0.4486 \frac{\mathrm{Ns}}{\mathrm{m}}, c_{d}^{0}=0.5089 \frac{\mathrm{Ns}}{\mathrm{m}}\right)$. MATLAB is used to simulate the simplified model.

The force, $F$, given to the shaker is a half-sine input such that the peak acceleration of the shaker is $100 \mathrm{G}$. Three shock durations are used in the simulation, they are 0.5 $\mathrm{ms}, 1 \mathrm{~ms}$, and $2 \mathrm{~ms}$. The $\delta_{a d}$ of the unmodified configuration are then compared with the other two cases: (i) the case where the damping factor of the baseplate $\left(c_{b}\right)$ is increased and (ii) the case where the damping factor of the arm $\left(c_{a}\right)$ is increased. The case where the damping factor of the disk is increased is not performed since it is unlikely that we can modify the damping factor of the disk in practice. The value of $\delta_{a d}$ which defines failure of the HDD is not studied in this paper, and this section focuses on the study of reducing $\delta_{a d}$ by increasing the damping factor of the baseplate and the arm.

Figures 3, 4, and 5 show the arm-disk relative displacement response for three shock durations $(0.5 \mathrm{~ms}, 1 \mathrm{~ms}$, and $2 \mathrm{~ms})$. We can see from Figs. 3, 4, and 5 that increasing the damping factor of the baseplate can effectively reduce the relative arm-disk displacement response compared to increasing the damping factor of the arm only. These results can be explained as follows:

As we have discussed in the subsection "Theoretical analysis", increasing $c_{a}$ only will only increase the damping ratio of the arm mode. Since the response of the disk is not affected by the increase of $c_{a}$, the arm and disk response could be more out of phase compared to the unmodified configuration. Out of phase here means that the arm and disk are moving in different directions so that their relative displacement becomes larger. We can see from Figs. 3, 4, and 5 that at some points the arm-disk relative displacement response is larger than the unmodified configuration. On the other hand, by increasing $c_{b}$, the damping ratio of all modes are increased. The $\delta_{a d}$ is reduced, as expected from the discussion in the subsection "Theoretical analysis".

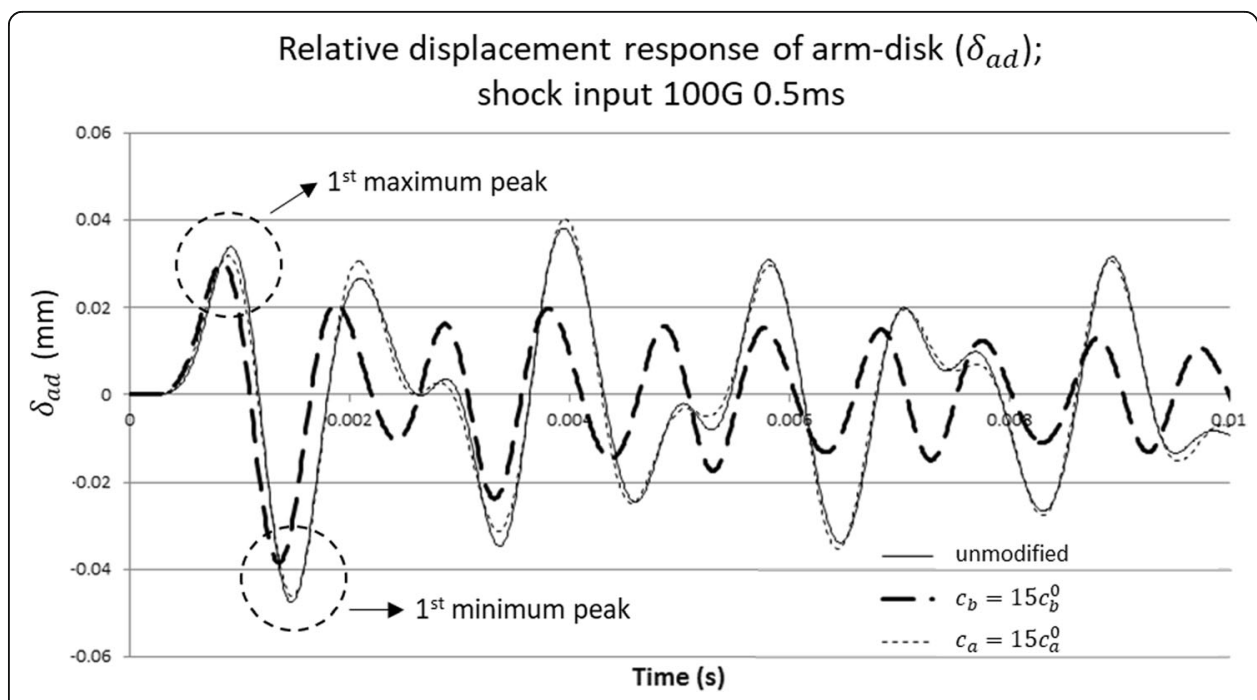

Fig. $3 \delta_{a d}$ response, HDD subjected to shock input $100 \mathrm{G} 0.5 \mathrm{~ms}$ 


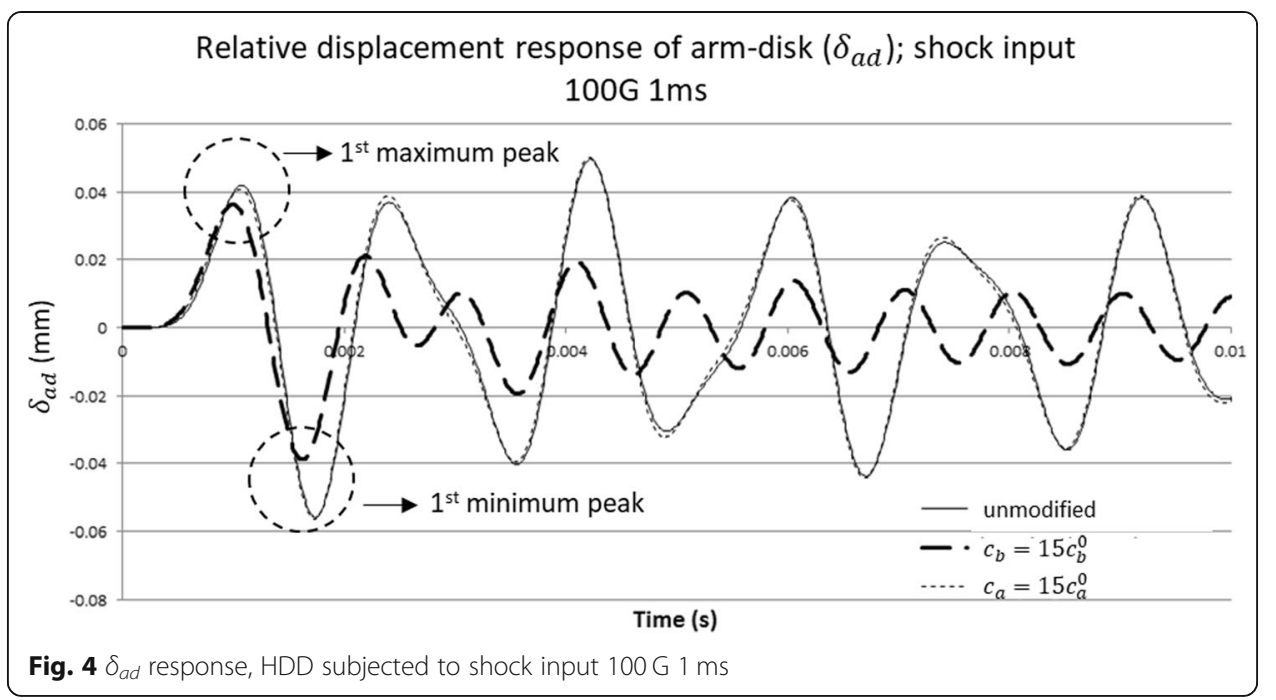

The optimum damping factor increase of the baseplate is also investigated. The damping factor of the baseplate is increased incrementally from $c_{b}^{0}$ up to $70 c_{b}^{0}$, and the range between the 1st maximum peak and the 1st minimum peak of the relative armdisk displacement response over time is measured for three shock duration cases. Let $\left(\delta^{\max }-\delta^{\min }\right)$ denotes the difference between the 1st maximum peak and the 1st minimum peak. Let also $\left(\delta^{\max }-\delta^{\min }\right)^{0}$ denotes the difference between the 1st maximum peak and the 1 st minimum peak when $c_{b}=c_{b}^{0}$. Figure 6 plots $\frac{\left(\delta^{\max }-\delta^{\min }\right)}{\left(\delta^{\max }-\delta^{\min }\right)^{0}} \times 100 \%$ versus $\beta$, where $\beta$ is the damping factor multiplier, i.e., $c_{b}=\beta c_{b}^{0}$.

From Fig. 6, it can be observed that the optimum damping factor for three shock duration cases is around 25 times of the unmodified value. The $100 \%$ displacement range is when the baseplate damping factor is set to be equal to $c_{b}^{0}$ or $\beta=1$. From Fig. 6, if we use more than 25 times of $c_{b}^{0}$, the improvement for $0.5 \mathrm{~ms}$ shock duration starts to decrease. While for $1 \mathrm{~ms}$ shock duration, when we use more than 25 times of $c_{b}^{0}$, the shock resistance improvement becomes less and less significant. This case is different

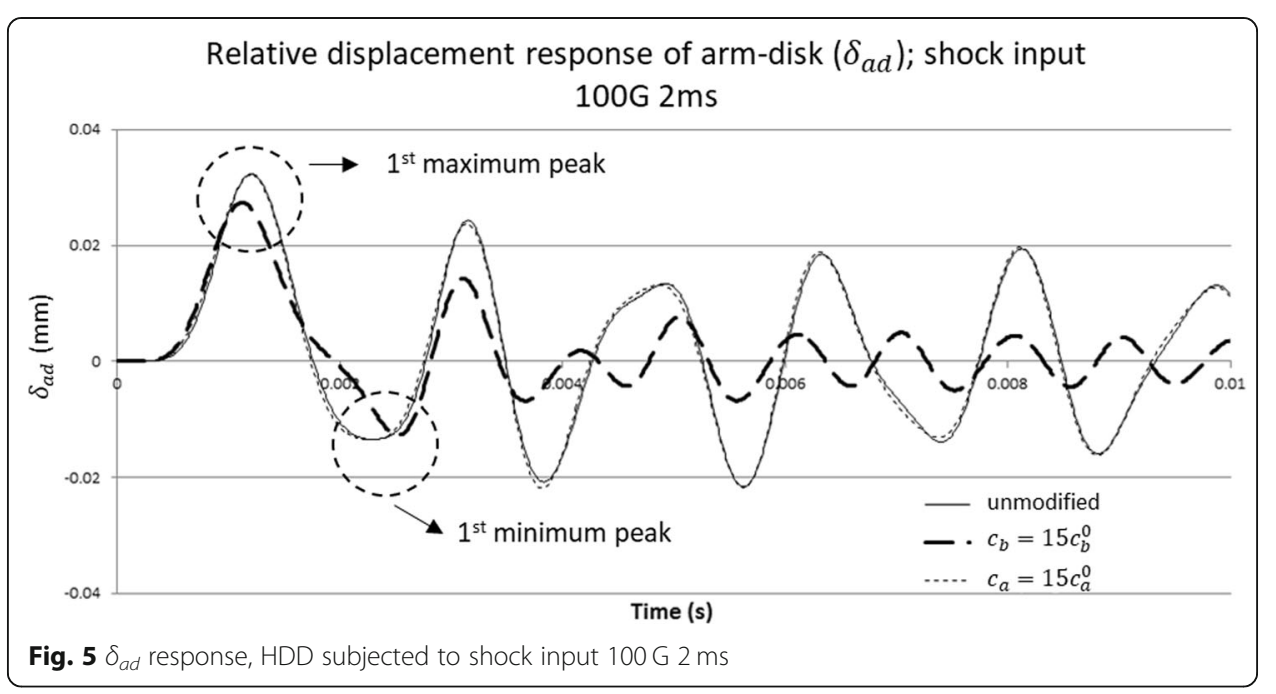




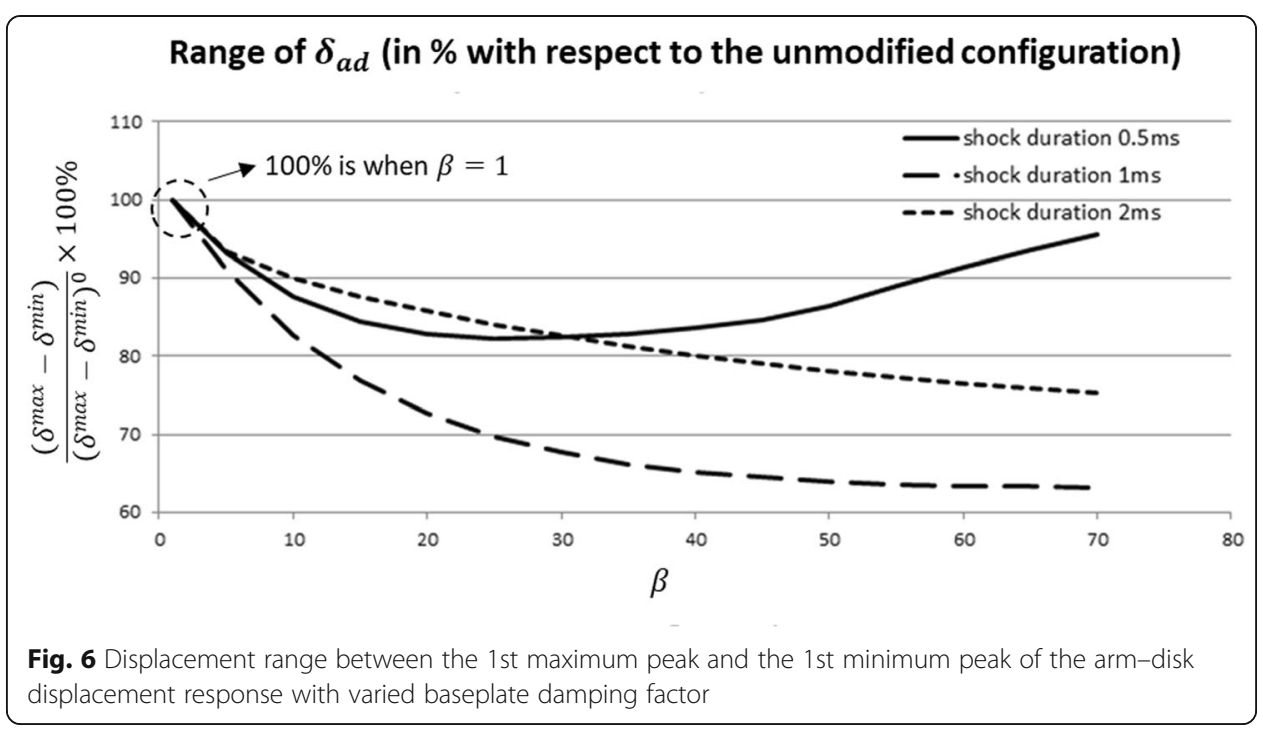

for the 2-ms shock duration: the shock resistance improvement is increasing almost linearly with the increase in the damping factor of the baseplate.

The above phenomenon can be explained by examining the transmissibility curve for a single degree of freedom with base excitation (see [22]). From the transmissibility curve, when the excitation frequency is close to the natural frequency of the system, a relatively high damping ratio is very effective to reduce the transmissibility. However, when the excitation frequency is higher than the natural frequency of the system, a system with a high damping ratio has higher transmissibility than the system with a low damping ratio. Thus, if we want to reduce the displacement on base excitation problem, when the excitation frequency is higher than the natural frequency, we should choose a relatively a low damping ratio.

The baseplate's natural frequency is around $600-800 \mathrm{~Hz}$, which makes the baseplate mode to be excited by all shock duration of $2 \mathrm{~ms}, 1 \mathrm{~ms}$, and $0.5 \mathrm{~ms}$ (see the FFT of the shock inputs in Fig. 7).

For the shock duration of $2 \mathrm{~ms}$, the FFT shows that the dominant excitation frequency range of the shock input is close to the baseplate's natural frequency. This is the reason that the improvement curve for the shock duration $2 \mathrm{~ms}$ of Fig. 5 keeps increasing when we increase the damping value of the baseplate. For the shock duration of $1 \mathrm{~ms}$, the shock excitation frequency range is a little bit higher than the baseplate's natural frequency, and thus high damping value of the baseplate (when $\beta>25$ in Fig. 6) is not effective to reduce the arm-disk relative displacement response. Lastly, for the shock duration of $0.5 \mathrm{~ms}$, the shock excitation frequency range is much higher than the baseplate's natural frequency. As a result, the high damping value of the baseplate (when $\beta>25$ in Fig. 6) yields in the lower improvement in the arm-disk displacement response.

Remark: the label high and low damping ratio in the above discussion is concluded solely from the results in Fig. 6.

\section{Non-operating shock experiments}

The subsection "Simulation of the simplified baseplate-HAA-disk model" indicates that increasing the damping factor of the baseplate results in the reduction of arm-disk 


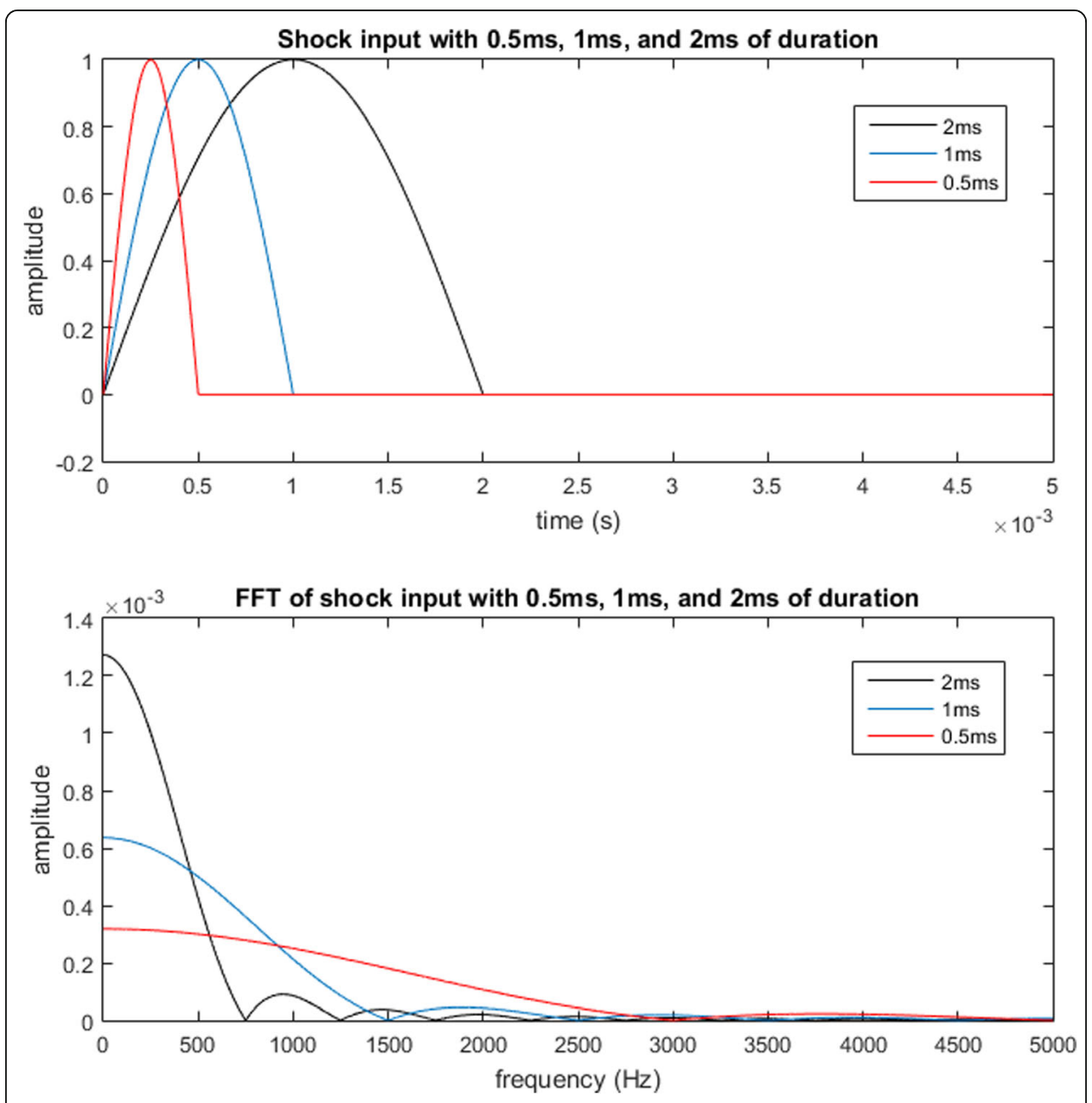

Fig. 7 FFT of shock inputs with duration of $0.5 \mathrm{~ms}, 1 \mathrm{~ms}$, and $2 \mathrm{~ms}$ generated using MATLAB

relative displacement or $\delta_{a d}$ when the HDD is subjected to shock input. In this subsection, we apply the same method as in the subsection "Simulation of the simplified baseplate-HAA-disk model" to a real 2.5" HDD which is to increase the damping factor of the baseplate, and then, we perform the non-operating shock tests to find out how much reduction to $\delta_{a d}$ that can be obtained on the real 2.5" HDD. There are two specific things we first need to answer in performing the non-operating shock tests:

(i) The method in increasing the damping factor of the baseplate.

(ii) The location on the baseplate where we should increase its damping factor.

In answering the first point, we chose a dampening layer from manufacturer $3 \mathrm{M}$ which has low outgassing property. The dampening layer material is based on polymer (see [23]). This property is important so that the dampening layer does not contaminate the internal environment of the HDDs. The thickness of the dampening layer is $0.05 \mathrm{~mm}$ and it is easy to be applied to the baseplate since it is working like a tape. The dampening layer used in the experiment is given in Fig. 8.

To answer the second point, a non-op-shock tests were performed. Referring to the discussion in the subsection "Simulation of the simplified baseplate-HAA-disk model", 
the non-op-shock tests use the arm-disk relative displacement as the improvement indicator. Several configurations of dampening layer placement on the baseplate were tested and the arm-disk relative displacement was monitored. The best dampening layer configuration from non-op-shock test is then used in HDDs for op-shock tests. In both non-operating and op-shock tests, commercial 2.5" HDDs are used. While the non-op-shock tests were done to find out the optimum dampening layer configuration, the op-shock tests were done to find out the op-shock tolerance improvement by using the optimum dampening layer configuration.

We note that the HDDs used in the experiments use single stage actuator. In the interest of the result from the subsection "Simulation of the simplified baseplateHAA-disk model", where the shock duration $0.5 \mathrm{~ms}$ has an optimum point with the lowest $\beta$, the non-operating and op-shock tests are carried out using $0.5 \mathrm{~ms}$ shock duration.

The non-op-shock tests involve the use of a shock tower to simulate the HDDs being dropped to the floor. The experiment set up is shown in Fig. 9. The shock tower has a guide-pole that holds the shock table such that the shock table can be dropped into the base and keeping the HDD facing in one direction during the drop test. The drop height can be adjusted to adjust the shock magnitude (the G level), while a soft material such as Delrin (a kind of plastic) can be placed on the drop area to adjust the shock duration. The shock magnitude and duration are adjusted and confirmed by using accelerometer attached on the shock table. To measure the $\delta_{a d}$, that is the relative displacement between the arm and the disk, a laser Doppler interferometer is used. The laser Doppler has two probes, where laser probe A shone the arm tip and laser probe B shone the outer disk point. The output from the laser Doppler interferometer is the relative displacement measured by the two probes and the initial measurement is normalized to zero. The output from the accelerometer and the laser Doppler interferometer are routed to the dynamic signal analyzer for recording purpose.

As we can see in Fig. 9, the HDD is tested in parking condition. To sustain the structural stiffness of the HDD during shock tests, the top cover of the HDD is still used, but the top cover is partially cut on the area where the lasers are being pointed (not shown in Fig. 9). It is worth noting that a non-op-shock test by positioning the arm-tip on top of the outer-disk is not possible since the laser can only point to the arm tip in

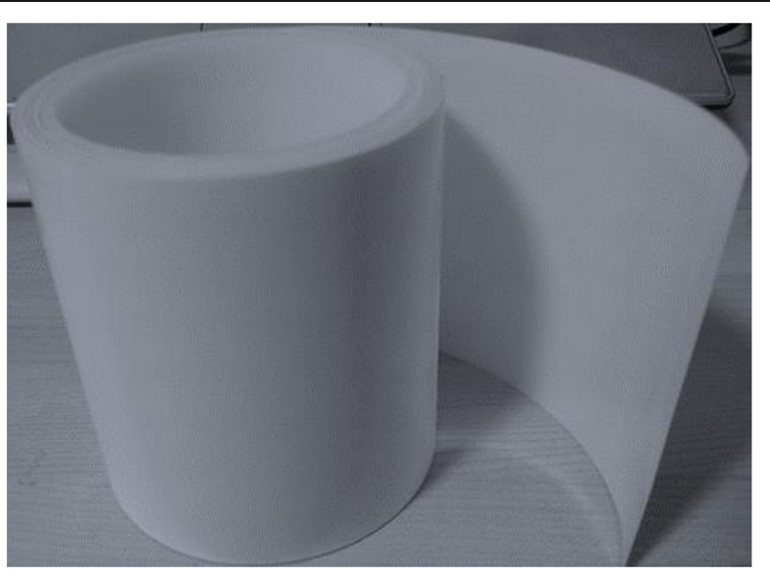

Fig. 8 Dampening layer 


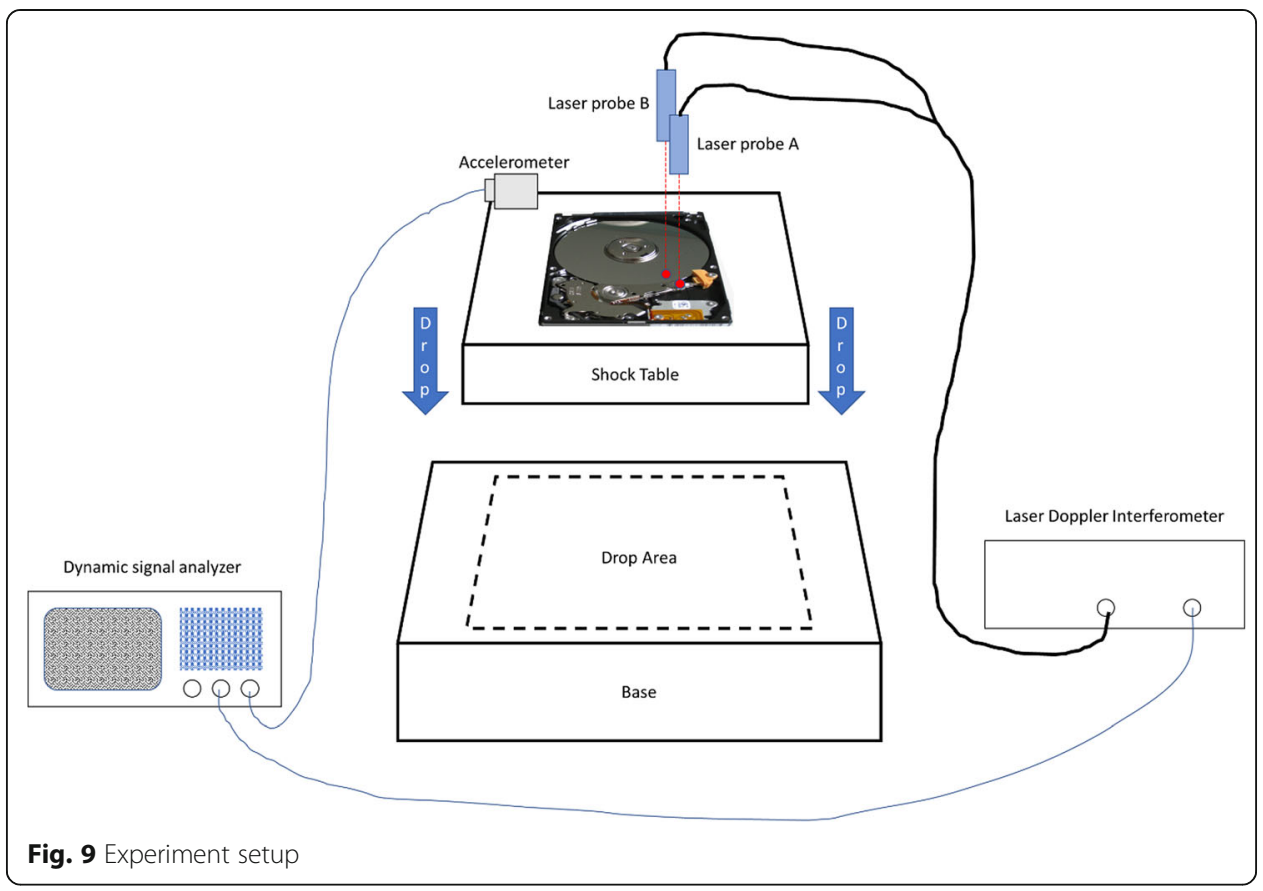

that situation. In addition, since the suspension's stiffness is relatively low, the arm bending mode is not affected significantly by the parking position.

To find suitable areas on the baseplate to apply the dampening layer, we refer to the discussion in the subsection "Theoretical analysis" where the damping factor increase on the baseplate is meant to increase the damping ratio of the baseplate's 1st bending mode, disk's 1st bending mode, and the arm's 1st bending mode. We denote these modes as the low frequency modes. Thus, the dampening layer must be placed on baseplate's areas that has high strain on these low frequency modes. To this end, we perform a modal analysis on the finite element model (FEM) of the HDD being used in the experiment. The modal analysis is done using finite element analysis package, ANSYS.

Figure 10 shows the finite element model of the baseplate, where the four corners A, $\mathrm{B}, \mathrm{C}$, and $\mathrm{D}$ are constrained in all direction for the modal analysis. The modal analysis is done on the full finite element model of the HDD, but in Fig. 10 we only select the baseplate elements to show the strain measurement locations and the first bending mode of the baseplate. The first bending mode of the baseplate of our HDD model is $729 \mathrm{~Hz}$ which is within the range of $600-800 \mathrm{~Hz}$. The strain between two adjacent nodes are done on 6 selected lines as shown in Fig. 10. To measure the strain, the eigenvector along the line for several modes is taken from the modal analysis result. Then, the strain is calculated as the difference between the eigenvector element between two adjacent nodes. This strain is a relative strain, and not to be confused with absolute strain measurement related to stress when the structure is being loaded. The strain measurement on the 6 lines is shown in Fig. 11.

From Fig. 11, the first three lowest frequencies are the 1st bending mode of the baseplate, disk, and arm, respectively. The high or low strain is determined by comparing the relative strain on the mode, and not to be compared with the other mode. For example, at line 1, Fig. 11 shows that the mid points of line 1 has high strain for the 729 

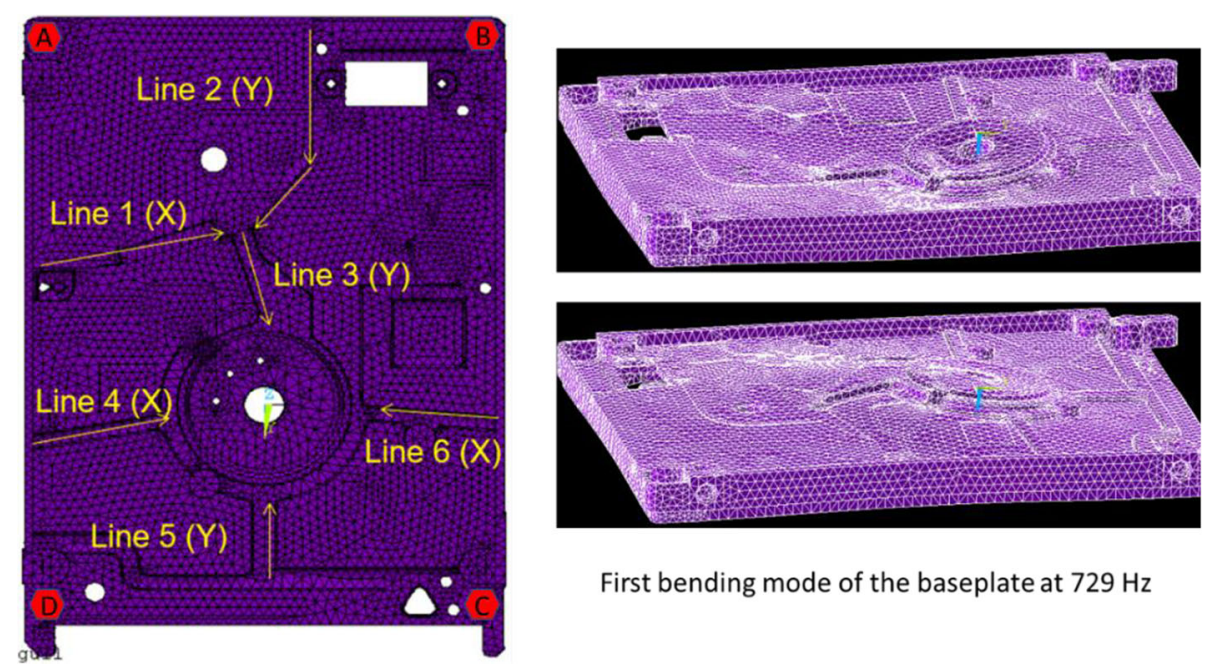

First bending mode of the baseplate at $729 \mathrm{~Hz}$

Fig. 10 Boundary condition on baseplate, strain measurement location, and first bending mode of the baseplate at $729 \mathrm{~Hz}$

$\mathrm{Hz}, 957 \mathrm{~Hz}$, and the $1551 \mathrm{~Hz}$. Meanwhile, for $1731 \mathrm{~Hz}$, the leftmost, mid, and rightmost points have comparable strain. Since the low frequency modes have the highest strain at the mid points, we mark the mid points of line 1 as having high strain for low frequency modes, while the leftmost and the rightmost points of line 1 have high strain for high frequency modes. The information from Fig. 11 is used as initial guess to apply the dampening layer on the back of the baseplate. The leftmost picture of Fig. 12 is the first configuration that we try. From there, we performed several trial and error until we find the most optimum dampening layer configuration.

The dampening layer is applied to the backside of the baseplate by first removing the PCB. Figure 12 shows a sample of dampening layer placement configuration which was tested during the non-op-shock tests. Only one HDD is used in the non-op-shock tests, which means that the new damping layer is placed by first removing the previously tested dampening layer. This is done to eliminate differences between different HDD batches. For each HDD, 10 drop tests are done, and the results are averaged.

In this paper, we show the results from the optimum dampening layer configuration only. The optimum dampening layer configuration is shown in Fig. 13. By optimum, we mean the configuration which results in the highest reduction of the peak of the arm-disk relative displacement. The optimum dampening layer placement that we found on this HDD is on the edge of the disk-spindle area and below the HAA's pivot. It is noted that the same dampening layer configuration might not work on different types of HDDs, since they will have different 1st bending mode of the baseplate.

Figure 14 shows the relative arm-disk displacement response over time obtained from the experiment. The initial value of the measurement is zero since the relative displacement measured by the laser Doppler interferometer is normalized at zero. The results from both the unmodified and HDD with dampening layer are plotted on this figure. The shock level used in the experiment was $100 \mathrm{G} 0.5 \mathrm{~ms}$. From Fig. 14, the $\frac{\left(\delta^{\text {max }}-\delta^{\text {min }}\right)}{\left(\delta^{\text {max }}-\delta^{\text {min }}\right)^{0}} \times 100 \%$ is around $80 \%$. This number $(80 \%)$ is close to the result in subsection 2.2 where damping factor of baseplate is set to 25 times of the unmodified value. 
Meanwhile, other dampening layer configurations shown in Fig. 12 have $\frac{\left(\delta^{\max }-\delta^{\min }\right)}{\left(\delta^{\max }-\delta^{\min }\right)^{0}} \times 10$ $0 \%$ of $88 \%, 92 \%, 94 \%$, and $91 \%$.

\section{Operating shock experiments}

In the op-shock experiments, two sets of HDDs were prepared. The HDDs used are of similar type with the one tested in the non-operating tests, and they have similar number of platter and capacity. The first set is two (2) unmodified HDDs (HDDs without dampening layer), and the second set is three (3) HDDs with the dampening layer on the baseplate (the dampening layer placement configuration is the one shown in Fig. 13).

Like the non-op-shock test, a shock duration of $0.5 \mathrm{~ms}$ was used. As discussed in the previous subsection, a shock duration of $0.5 \mathrm{~ms}$ has the least room for improvement in terms of non-op-shock tolerance, and therefore, it will be our interest to find out the op-shock resistance improvement for $0.5-\mathrm{ms}$ shock duration. The two sets of HDDs were shock-tested using the same shock table as used in the non-op-shock test. In this op-shock test, the G level is increased incrementally by $25 \mathrm{G}$ from $200 \mathrm{G}$ until all HDD fail. The shock duration is kept the same $(0.5 \mathrm{~ms})$ for all op-shock tests. The op-shock test results are given in Table 1.

The failure indicator in the op-shock test is whether the read/write head touches/ slaps the disk. To retain the same op-shock test condition for all HDDs, a routine was

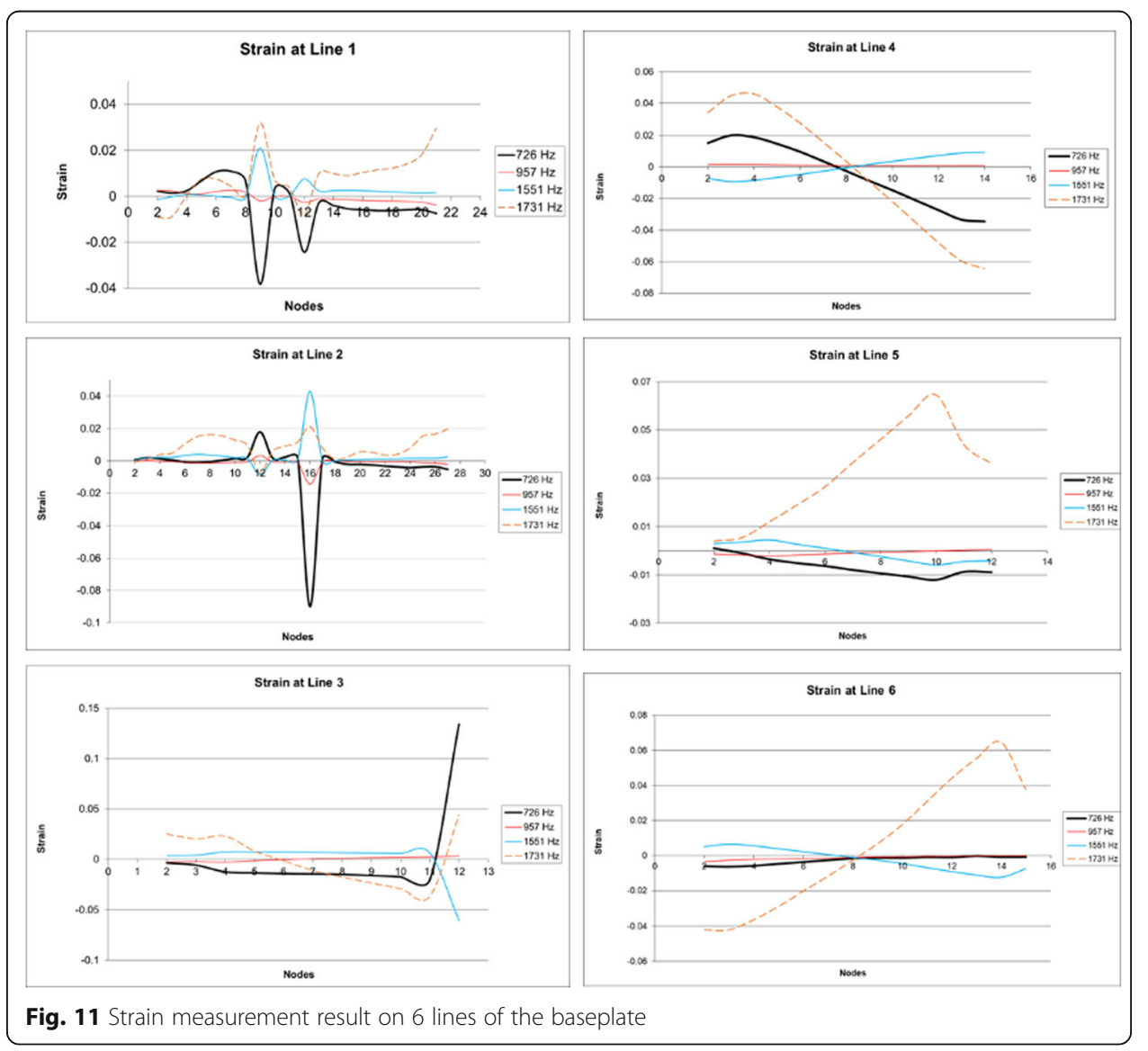




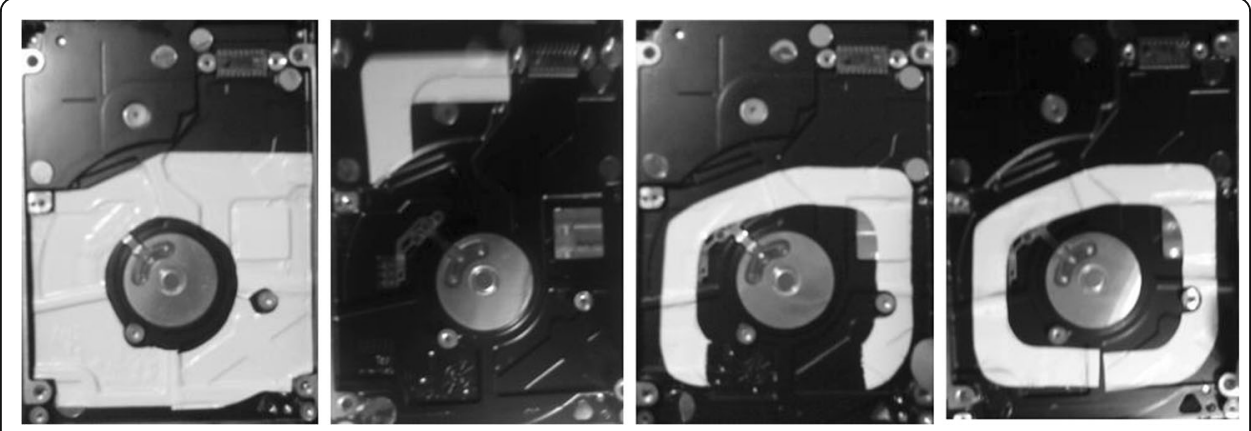

Fig. 12 Samples of dampening layer configurations tested on the non-op-shock tests; the white tape is the dampening layer

run in each op-shock test to position the actuator arm in the outer disk diameter (OD) position. A program was run to scan and check the disk for the bad sector before and after the op-shock test. The number of bad sectors before and after op-shock tests is then compared. If the bad sector was found increasing after the op-shock test, this means the disk was damaged in some places due to head slap when the HDD is subjected to the shock, and thus the HDD fails the op-shock test.

From the results of Table 1, the unmodified HDD starts to fail at $275 \mathrm{G} 0.5 \mathrm{~ms}$, while for the HDD with dampening layer; they all fail at $325 \mathrm{G} 0.5 \mathrm{~ms}$. This shows a $50 \mathrm{G}$ advantage of the HDDs with the dampening layer compared to the unmodified HDDs. The $50 \mathrm{G}$ advantage of the HDD with the dampening layer can be explained as follows.

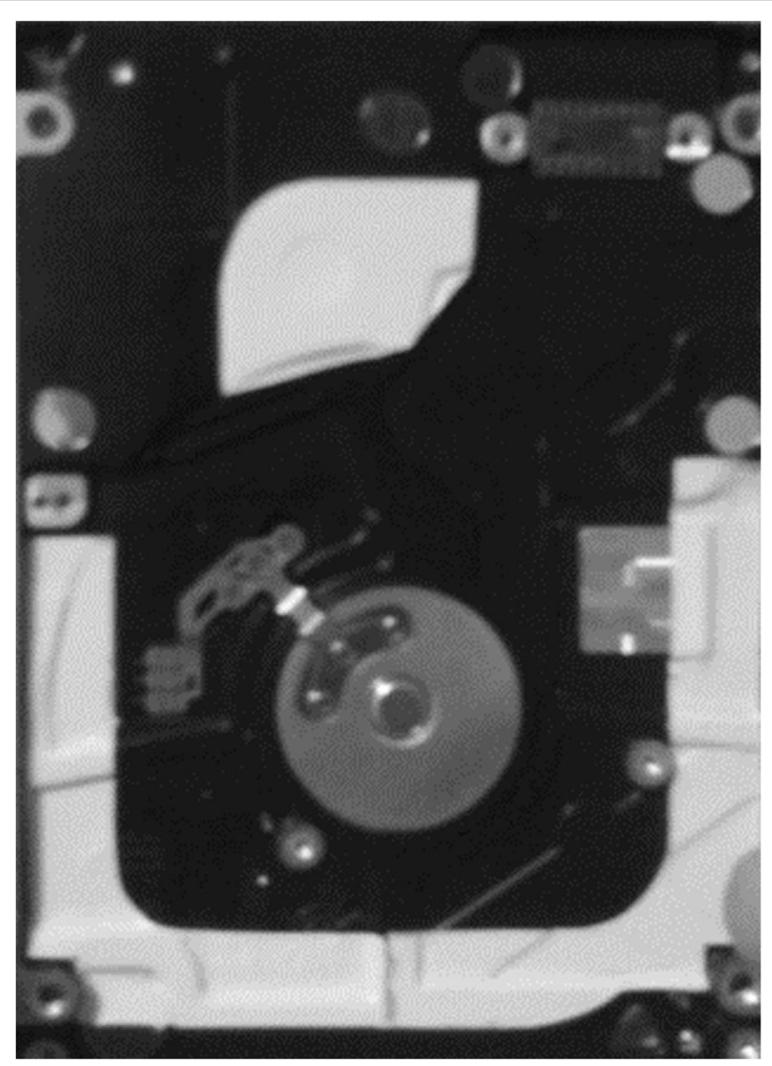

Fig. 13 Optimum dampening layer placement during non-op-shock tests 
From the non-op-shock tests, we know that the HDD with the dampening layer has lower arm-disk relative displacement (experiments are done by applying the same shock level to HDD with and without dampening layer). This also means that a higher shock level is needed so that the HDD with dampening layer has similar arm-disk relative displacement. Here, we refer to the discussion in the "Methods" section in which the arm-disk relative displacement is strongly related to the head-disk failure mechanism.

The op-shock resistance improvement from this experiment is $18.18 \%\left(\frac{50}{275} \times 100 \%\right)$. Although this number is close to the improvement found in the non-op-shock test results (relative arm-disk displacement reduction of $80 \%$ or improvement of $20 \%$ ) and MATLAB simulation on the simplified model done in the subsection "Simulation of the simplified baseplate-HAA-disk model", it is difficult to take precise conclusion on the percentage of improvement since the increment of the op-shock test level that can be done is $25 \mathrm{G}$. However, we can conclude that, qualitatively, the improvement found in the non-op-shock tests is also found on the op-shock tests.

\section{Conclusion}

A strategy to apply dampening layer on the baseplate of the HDD to increase its opshock tolerance has been presented in this paper. Technical analysis and simulation on the simplified model of baseplate-HAA-disk suggest that increasing the damping factor of the baseplate has a potential to improve the op-shock tolerance of the HDD. This improvement is implied by the reduction in the arm-disk relative displacement when the HDD is subjected to shock. The findings from the technical analysis and simulation on the simplified model are verified by the non-op-shock tests on the real HDD. Finally, op-shock tests were done to test the hypothesis, and the results from the tests show that HDDs with dampening layer have higher op-shock resistance compared to HDDs without the dampening layer.

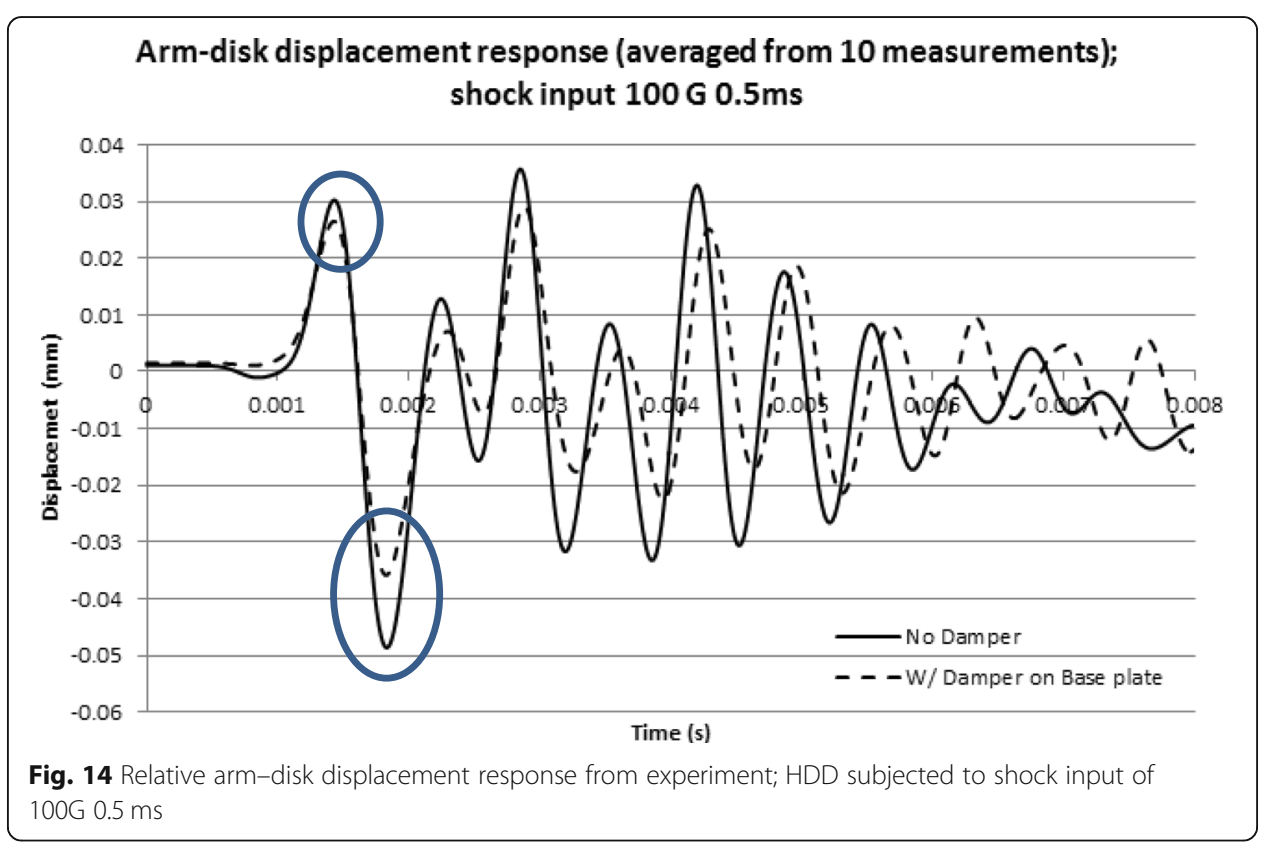


Table 1 Op-shock test results of unmodified HDD and HDD with dampening layer on the baseplate (all shock is of 0.5 -ms duration)

\begin{tabular}{lllllll}
\hline & 200 G & 225 G & 250 G & 275 G & 300 G & 325 G \\
\hline Unmodified HDD & & & & & & \\
HDD \#1 & Pass & Pass & Pass & Fail & Fail & Fail \\
HDD \#2 & Pass & Pass & Pass & Pass & Fail & Fail \\
HDD with dampening layer & & & & & \\
HDD \#4 & Pass & Pass & Pass & Pass & Pass & Fail \\
HDD \#5 & Pass & Pass & Pass & Pass & Pass & Fail \\
HDD \#6 & Pass & Pass & Pass & Pass & Pass & Fail \\
\hline
\end{tabular}

In this work, we found that the dampening layer cannot be placed arbitrarily. The dampening layer must be placed on areas so that the 1st bending mode of the baseplate, disk, and arm are dampened out. The dampening layer placement is initiated by modal analysis to obtain an initial guess of the area. After that, a non-op-shock tests are done to fine-tune the area.

One of the problems that has not been addressed by this paper is to devise a method to generalize the solution for different HDDs. With the proposed method, different HDDs need different sets of experiments and thus it is not practical for a mass production system. Another issue is that thermal insulation due to the application of the dampening layer has not been addressed yet in this study. These problems will be discussed in our future study.

\section{Abbreviations}

HDD: Hard disk drive; HAA: Head actuator assembly; FEM: Finite element model; OD: Outer disk; PCB: Printed circuit board

\section{Acknowledgements}

The authors would like to acknowledge the support from Indonesia Endowment Fund for Education (LPDP) and Center of Research and Community Service (CRCS) of Sampoerna University.

\section{Authors' contributions}

D.W.D. conceived and designed the simulation and experiments, performed the simulation and experiments, analyzed and interpreted the data, wrote the original paper, and wrote the revised manuscript. D.W.D., F.F.Y., B.A.B., and F.T. read and approved the final manuscript.

\section{Funding}

This research is funded by the Indonesia Endowment Fund for Education (LPDP) under Research and Innovation Program (RISPRO) for electric vehicle development with contract no. PRJ-85/LPDP/2020 and Center of Research and Community Service (CRCS) of Sampoerna University.

Availability of data and materials

All data are available from the authors.

\section{Declarations}

Competing interests

The authors declare that they have no competing interests.

\section{Author details}

${ }^{1}$ Mechanical Engineering Study Program, Sampoerna University, Jakarta, Indonesia. ${ }^{2}$ School of Mechanical and Aerospace Engineering, Nanyang Technological University, Singapore, Singapore. ${ }^{3}$ Faculty of Mechanical and Aerospace Engineering, Institut Teknologi Bandung, Bandung, Indonesia. 
Received: 3 September 2021 Accepted: 16 December 2021

Published online: 28 January 2022

\section{References}

1. Suzuki K, Maeda R, Chu J, Kato T, Kurita M (2003) An active head slider using a piezoelectric cantilever for in situ flyingheight control. IEEE Trans Magn 39(2 I). https://doi.org/10.1109/TMAG.2003.808934

2. Hong EJ, Kim WS, Ho SL (2006) Design modification of micro-actuator to improve shock resistance of HDD. https://doi. org/10.1109/APMRC.2006.365928

3. Marchon B, Pitchford T, Hsia YT, Gangopadhyay S (2013) The head-disk interface roadmap to an areal density of 4 Tbit/ in 2. Adv Tribol. https://doi.org/10.1155/2013/521086

4. Jayson EM, Murphy J, Smith PW, Talke FE (2003) Effects of air bearing stiffness on a hard disk drive subject to shock and vibration. J Tribol 125(2). https://doi.org/10.1115/1.1509770

5. Li L, Bogy DB (2014) Operational shock failure mechanisms in hard disk drives. J Tribol 136(3). https://doi.org/10.1115/1.4 027209

6. Lin CC (2002) Finite element analysis of a computer hard disk drive under shock. J Mech Des Trans ASME 124(1). https:// doi.org/10.1115/1.1424299

7. Shengkai $Y$, Jianqiang M, Wei H, Weidong Z, Chin TC (2016) Flexible support for hard disk drives to enhance operational shock resistance. https://doi.org/10.1109/APMRC.2016.7524284

8. Luo J, Shu DW, Shi BJ, Gu B (2007) The pulse width effect on the shock response of the hard disk drive. Int J Impact Eng 34(8). https://doi.org/10.1016/j.ijimpeng.2006.07.005

9. Nicholson JW, Hobbet JR, Jakes PJ. Systems and methods for protecting hard disk drives. Singapore: Lenovo; 2020 https:/patft.uspto.gov/netacgi/nph-Parser?Sect1=PTO1\&Sect2=HITOFF\&d=PALL\&p=1\&u=\%2Fnetahtml\%2FPTO\%2 Fsrchnum.htm\& $r=1 \& f=G \& l=50 \& s 1=10,741,209 . P N \cdot \& O S=P N / 10,741,209 \& R S=P N / 10,741,209$

10. HwaLiang Ng, Camalig CJB, Buang A. Head-slap mitigation in a data storage device. 2012. https://patents.google.com/ patent/US8089733B2/en.

11. Zhu DL, Wang AL, Jiang T (2006) Topology design to improve HDD suspension dynamic characteristics. Struct Multidiscip Optim 31(6). https://doi.org/10.1007/s00158-005-0581-6

12. Lim S, Chang YB, Park NC, Park YP (2006) Optimal design of rubber mounts supporting notebook hdd for shock and vibration isolation. https://doi.org/10.1109/APMRC.2006.365930

13. Djamari DW (2010) Mitigating shock of operating HDDs using smart suspensions. Singapore: Nanyang Technological University

14. Yap FF, Vahdati N, Harmoko H (2006) Design and analysis of vibration isolation systems for hard disk drives. J Magn Magn Mater 303(2 SPEC. ISS). https://doi.org/10.1016/j.jmmm.2006.01.114

15. Harmoko H, Yap FF, Vahdati N, Li C (2009) Design and analysis of shock and random vibration isolation of operating hard disk drive in harsh environment. Shock Vib 16(2). https://doi.org/10.1155/2009/959714

16. Eguchi T, Tomida K, Okazaki T. Hard disk drive with a vibration isolation frame. 2018.

17. Schwager MA, Miller MT. Suspended hard disk drive system for portable computers. 2017. https://patents.google.com/ patent/US9778701.

18. Sezgen HC, Tinkir M (2021) Optimization of torsional vibration damper of cranktrain system using a hybrid damping approach. Eng Sci Technol Int J 24(4). https://doi.org/10.1016/j.jestch.2021.02.008

19. Biglari H, Golmohammadi M, Hayati S, Hemmati S (2021) Vibration reduction of a flexible robot link using a frictional damper. JVC/Journal Vib Control 27(9-10). https://doi.org/10.1177/1077546320936092

20. Jang GH, Seo CH, Lee HS (2007) Finite element modal analysis of an HDD considering the flexibility of spinning diskspindle, head-suspension-actuator and supporting structure. Microsyst Technol 13(8-10). https://doi.org/10.1007/s00542006-0276-y

21. Adams V, Askenazi A (1999) Building better products with finite element analysis. OnWord Press

22. Meirovitch L. Fundamentals of vibrations. McGraw-Hill Professional; 2003.

23. 3M, "3M Ultra-Pure Viscoelastic Damping Polymer 242NR02." p. 4, 2012. https://multimedia.3m.com/mws/media/3 079790/3mtm-ultra-pure-viscoelastics-damping-polymer-242nr02.pdf

\section{Publisher's Note}

Springer Nature remains neutral with regard to jurisdictional claims in published maps and institutional affiliations.

\section{Submit your manuscript to a SpringerOpen ${ }^{\circ}$ journal and benefit from:}

- Convenient online submission

- Rigorous peer review

- Open access: articles freely available online

High visibility within the field

- Retaining the copyright to your article

Submit your next manuscript at $\boldsymbol{s p r i n g e r o p e n . c o m ~}$ 